\title{
Antoine Missemer - Nicholas Georgescu-Roegen, pour une révolution bioéconomique suivi de De la science économique à la bioéconomie de Nicholas Georgescu-Roegen - Lyon, ENS Éditions, coll. « Feuillets », 2013, 136 p.
}

Jean-Marie Harribey

\section{(2) OpenEdition}

Electronic version

URL: http://journals.openedition.org/economierurale/4413

DOI: $10.4000 /$ economierurale. 4413

ISSN: 2105-2581

\section{Publisher}

Société Française d'Économie Rurale (SFER)

Printed version

Date of publication: 15 July 2014

Number of pages: 125-130

ISSN: 0013-0559

\section{Electronic reference}

Jean-Marie Harribey, « Antoine Missemer - Nicholas Georgescu-Roegen, pour une révolution bioéconomique suivi de De la science économique à la bioéconomie de Nicholas Georgescu-Roegen Lyon, ENS Éditions, coll. « Feuillets », 2013, 136 p. », Économie rurale [En ligne], 342 | juillet-août 2014, mis en ligne le 15 juillet 2014, consulté le 22 septembre 2020. URL : http://journals.openedition.org/ economierurale/4413; DOI : https://doi.org/10.4000/economierurale.4413 
Antoine MISSEMER

\section{Nicholas Georgescu-Roegen, pour une révolution bioéconomique}

\section{Suivi de De la science économique à la bioéconomie de Nicholas Georgescu-Roegen}

Lyon, ENS, coll. « Feuillets », 2013, 136 p.

\section{À la (re)découverte de Georgescu-Roegen}

es économistes qui s'intéressent vraiment à l'écologie, les écologistes, des plus modérés aux plus radicaux, les physiciens spécialistes de la thermodynamique, tous connaissent l'apport de Nicholas Georgescu-Roegen (1906-1994), ou en ont au moins entendu parler. Cet économiste roumain fut un perpétuel émigré à la recherche d'une nouvelle épistémologie de l'économie, un peu en GrandeBretagne et en France, beaucoup aux États-Unis où il connut Schumpeter, avec entre-temps un bref retour dans son pays d'origine. Connu de quelques chercheurs francophones parce qu'un recueil de ses articles fut traduit et publié par Jacques Grinevald et Ivo Rens en 1995 sous le titre La Décroissance, Entropie, écologie, économie $^{1}$, son œuvre méritait d'être plus largement diffusée alors que sévit une crise du capitalisme mondial dont la composante écologique est sans aucun doute maintenant majeure. Aussi, la publication en 2013 de l'ouvrage d'Antoine Missemer, jeune chercheur de l'Université de Lausanne, vient-elle combler une lacune. Et, disons-le d'emblée, avec beaucoup de brio, de clarté et de vue d'ensemble. C'est d'autant plus remarquable que I'œuvre de Georgescu-Roegen est difficile d'accès, à la mesure d'une part de la complexité des problèmes nés de l'insertion

1. Paris, Éditions Sang de la terre, 1995. Rééd. 2006. indépassable des activités humaines dans la biosphère, et aussi d'autre part à cause du renversement épistémologique et méthodologique de la discipline économique que Georgescu-Roegen propose.

Le livre de Missemer est composé de deux parties : dans la première, I'auteur présente la démarche et les propositions de Georgescu-Roegen, la seconde est une réédition d'un texte de synthèse de ce dernier, publié en 1978 dans la Revue $d^{\prime}$ 'économie politique ${ }^{2}$. L'ensemble est particulièrement utile pour saisir la très grande portée de cette œuvre et aussi peut-être les questions qui restent posées.

\section{L'application de la thermodynamique à l'économie}

Après quelques travaux de jeunesse de microéconomie, Georgescu-Roegen consacra l'essentiel de sa carrière à rebâtir une science économique en dehors de la mécanique newtonienne fondée sur des " raisonnements d'équilibre et de cycle ", nous dit Missemer : "Son regard doit se porter sur le cadre imposé par la thermodynamique qui a le mérite de décrire deux phénomènes particulièrement importants pour le monde économique : i) un processus, de quelque nature qu'il soit, implique toujours des changements qualitatifs, et ii) ces changements sont la plupart du temps irréversibles ${ }^{3}$. Selon Georgescu-Roegen, deux voies complémentaires s'ouvrent pour mieux comprendre le monde : la biologie évolutionniste et la physique thermodynamique qui permettent de fonder une épistémologie de la bioéconomie.

La biologie évolutionniste inscrit Georgescu-Roegen dans la lignée de

2. Georgescu-Roegen N. (1978). « De la science économique à la bioéconomie ». Revue d'économie politique, vol. 88, $\mathrm{n}^{\circ} 3$, mai-juin, p. 337-382. In Missemer A., (2013). Nicholas Georgescu-Roegen, pour une révolution bioéconomique. Lyon, ENS, coll. «Feuillets Économie politique moderne », 136 p.

3. Page 15 . 
Darwin et du mathématicien et physicien Alfred Lotka. II reprend de celui-ci la distinction entre instruments endosomatiques (moyens d'agir qui sont des constituants biologiques de l'individu) et instruments exosomatiques (outils). Comme les hommes utilisent de plus en plus ces derniers, qui proviennent toujours d'une activité économique, "la discipline économique apparaît nécessairement comme une extension de la biologie évolutionniste ${ }^{4} \mathrm{~d}$ 'où la dimension qualitative des processus économiques.

Mais c'est la proposition d'appliquer les principes de la thermodynamique à l'économie qui constitue l'originalité principale de Georgescu-Roegen. À la suite des travaux pionniers de Carnot et de Clausius au XIX siècle, les physiciens définissent quatre principes de la thermodynamique :

- Principe zéro : deux systèmes en équilibre thermique avec un troisième sont en équilibre thermique entre eux.

- Premier principe de conservation de la quantité d'énergie : l'énergie d'un système isolé reste constante. La conséquence est qu'on ne peut pas créer d'énergie, on ne peut que la transformer.

- Deuxième principe de dégradation de l'énergie en chaleur irrécupérable, dit loi de l'entropie qui crée une irréversibilité.

- Troisième principe de limite des possibilités de refroidissement d'un corps physique même si l'énergie nécessaire est disponible.

C'est la prise en compte de l'entropie dans les processus économiques qui, selon Georgescu-Roegen, doit bouleverser complètement les présentations habituelles de l'économie. Certes, la Terre n'est pas un système isolé puisqu'elle reçoit en permanence un flux d'énergie solaire, mais elle n'est pas non plus un système ouvert, car elle ne reçoit pas de matière du reste de l'univers. Elle est dans un cas intermédiaire dit fermé. C'est ce qui amènera

4. Page 20.
Georgescu-Roegen à considérer que le deuxième principe de l'entropie doit s'appliquer à l'énergie, mais aussi à la matière. En effet, même si l'énergie solaire permet aux processus vivants de se développer et de se complexifier, l'échelle de temps biologique et physique n'a rien de commun avec le temps humain et encore moins avec le temps économique. La rareté des ressources est donc une contrainte incontournable puisque l'activité économique conduit à dissiper la matière.

Il en résulte une impossibilité de recyclage total : impossibilité à la fois physique et logique puisqu'il subsiste toujours un déchet ultime, sauf à supposer un impossible recyclage immatériel. On retrouve l'idée qu'il n'y a pas de processus de production sans support matériel. Cependant, Missemer souligne que les physiciens ont observé " au niveau moléculaire des processus d'agrégation de matière dissipée à l'aide de grande quantité d'énergie libre $~^{5}$ venant affaiblir la portée de l'extension de la loi de l'entropie à la matière avancée par Georgescu-Roegen. C'est ce qui expliquerait en partie, selon Missemer, la difficulté pour GeorgescuRoegen de convaincre ses pairs des limites physiques à l'activité économique.

Georgescu-Roegen ne se contentait pas de cette investigation théorique, il s'avançait aussi sur le terrain programmatique. À ce titre, il proposait de « remplacer l'énergie terrestre par l'énergie solaire toutes les fois que cela est possible ", même s'il était conscient que, bien qu'elle soit la seule énergie libre, son intensité était faible et donc elle n'était pas encore "viable ${ }^{6}$. II proposait aussi de transformer toute l'agriculture en agriculture biologique et de définir ainsi la taille soutenable de la population mondiale souhaitable. Compte tenu de l'urgence écologique et des délais pour appliquer les nouveaux principes bioéconomiques, l'infléchissement des comportements économiques est nécessaire

5. Page 43 .

6. Pages 39,47 et 50 . 
dès maintenant ${ }^{7}$. On dirait aujourd'hui que la transition doit être amorcée rapidement. Pour cela, Georgescu-Roegen " milite pour deux types d'intervention publique ${ }^{8}$ : d'une part, réorienter les dépenses publiques pour favoriser un bon usage des ressources et aider les pays moins développés, et d'autre part réglementer l'activité économique de façon à inciter une production de biens durables et imposer si nécessaire des restrictions quantitatives à l'usage des ressources.

De tout cela, l'ouvrage de Missemer rend très bien compte dans ses deux premiers chapitres présentant la matrice théorique de Georgescu-Roegen et sa révolution bioéconomique. Le troisième chapitre surprendra peut-être certains auteurs ou militants qui se réclament de Georgescu-Roegen, car Missemer se livre à une entreprise de démystification salutaire des interprétations qui sont données de l'œurre de Georgescu-Roegen, voire des récupérations qui en sont faites. Ce chapitre intitulé « Halte à la (dé)croissance " examine les rapports que la théorie de Georgescu-Roegen entretient avec trois courants. D'abord le dialogue avec l'économie conventionnelle est qualifié de difficile par Missemer ; il s'est même soldé par un échec avec Solow et Stiglitz, deux des figures les plus éminentes du courant néoclassique. L'auteur souligne la faiblesse méthodologique de la fonction de production Cobb-Douglas, dénoncée par Georgescu-Roegen, car elle est bâtie sur l'hypothèse de substituabilité des facteurs de production?.

Avec le courant de l'économie écologique, bien représentée par la revue

\section{Page 50 .}

8. Id.

9. De manière totalement incohérente, nombre de théoriciens écologistes aujourd'hui utilisent cette fonction en pensant que l'introduction en son sein du facteur environnement la rend acceptable, ignorant qu'elle est fondée sur la substituabilité des facteurs entre eux. Pour une critique, voir Harribey J.-M. (2013). La richesse, la valeur et l'inestimable, Fondements d'une critique socioécologique de l'économie capitaliste, Paris, LLL.
Ecological Economics, Georgescu-Roegen a entretenu des rapports ambigus, estime Missemer. La raison en est sans doute, selon notre auteur, que, bien que partageant avec Georgescu-Roegen l'idée que les processus économiques sont en co-évolution avec les processus naturels, le courant de l'économie écologique est éclaté en plusieurs sous-courants plus ou moins radicaux ou plus ou moins en rupture avec l'approche conventionnelle, dont l'un des critères de démarcation est l'acceptation ou non du principe d'entropie de la matière. Au sujet du courant dit de la décroissance, Missemer montre qu'il ne peut véritablement se réclamer de GeorgescuRoegen et qu'il y a un " malentendu historique ${ }^{10}$ entre eux. La chose est d'autant plus compliquée à décortiquer qu'il existerait six familles d'écologistes : l'écologie profonde, le biorégionalisme, l'anarchoprimitivisme, l'écologie sociale, les décroissantistes et l'écologisme agrairien ${ }^{11}$. Seuls, les décroissantistes se réclament explicitement de Georgescu-Roegen. À l'appui de cette référence, il y a le fait que GeorgescuRoegen considérait que l'état stationnaire ne suffisait pas à résoudre le problème écologique et que « la croissance devait cesser, voire être renversée ". Cependant, note Missemer, il ne s'agirait pas de la croissance en soi, mais de la " croissance actuelle ". Georgescu-Roegen " ne dénonce pas la croissance comme dynamique générale de l'économie, mais la croissance telle qu'elle se manifeste dans les sociétés industrielles, c'est-à-dire à travers le prisme d'une prédation excessive des ressources naturelles [...] Ce n'est pas l'activité économique dans l'absolu qui doit être nécessairement remise en cause. Alors que la plupart des objecteurs de croissance estiment que la contingence historique a peu d'importance dans la problématique écologique, tant la croissance est intrinsèquement la source des problèmes environnementaux et sociaux rencontrés (Bayon et al., 2012 ${ }^{12}$; Latouche, 2011), interpréter ce célèbre
10. Page 67.
11. Id.
12. Pages 116-117. 
passage de Georgescu-Roegen comme un appui catégorique à la décroissance structurelle est sans nul doute le fruit d'un abus interprétatif $\aleph^{13}$. À juste titre, Missemer rapporte que Georgescu-Roegen tenait fermement à distinguer croissance et développement, contrairement au courant de la décroissance, point sur lequel " la filiation se trouve la plus fragilisée $»^{14}$. Enfin, l'auteur souligne que, pour GeorgescuRoegen, " en privilégiant l'énergie solaire en lieu et place des hydrocarbures, toute l'industrie énergétique devra changer de modèle, ce qui créera des baisses d'activité par ci, et des hausses d'activité par là ${ }^{15}$. D'où la conclusion sévère de Missemer à l'encontre des décroissantistes : " En réalité, en concentrant son attention sur les aspects quantitatifs de l'activité économique, et en refusant, pour certains de ses défenseurs, la notion de développement, la décroissance ne s'affranchit pas de l'épistémologie mécaniste. Elle ne peut, en ce sens, s'inscrire dans l'héritage bioéconomique. Bien entendu, même pour Georgescu-Roegen, I'application de mesures écologiques ambitieuses passera par la mise à l'index d'un certain nombre d'activités prédatrices ou polluantes, qui seront alors amenées à décroître. II ne s'agit pourtant pas d'ériger la décroissance comme principe premier et généralisé ${ }^{16}$.

\section{Une véritable rupture épistémologique?}

On dispose avec l'ouvrage synthétique de Missemer d'une très bonne introduction à l'œuvre de Georgescu-Roegen. À partir de

13. Page 70. L'auteur cite Bayon D., Flipo F., Schneider F. (2012), La décroissance : dix questions pour comprendre et débattre, Paris, La Découverte, $2^{e}$ éd ; et Latouche S. (2011), Vers une société frugale d'abondance, Paris, Mille et une nuits.

14. Page 73.

15. Page 72. Voir aussi p. 79. Missemer aurait pu citer un autre passage très célèbre de Georgescu-Roegen, La décroissance : entropie, écologie, économie, op. cit., p. 104-106, dans lequel il fait la distinction entre croissance et développement. 16. Page 79. là peut s'engager une discussion sur la portée épistémologique et méthodologique de cette œuvre. Tout d'abord, rompt-elle réellement avec l'économie standard néoclassique ? Missemer dit peut-être des choses un peu contradictoires: GeorgescuRoegen rompt-il avec ses premiers travaux microéconomiques dans le cadre néoclassique pour créer un cadre propre à la bioéconomie, ou bien y a-t-il une " cohérence d'ensemble $1{ }^{17}$ ? À quoi peut servir une théorie microéconomique de l'utilité alors qu'on sait de manière certaine qu'elle ne peut déboucher sur une théorie de l'utilité sociale d'ensemble ? Georgescu-Roegen abandonne certainement l'hypothèse de rationalité individuelle parfaite, mais cela ne résout pas la question de l'impossibilité d'agrégation des préférences et comportements individuels.

Parmi les facteurs de production, Georgescu-Roegen distingue les "facteurs-flux " et les " facteurs-fonds ". Missemer donne l'exemple du fournil du boulanger dans lequel il y a de la farine (facteur-flux) et le four (facteur-fonds). Quelle est l'innovation théorique par rapport aux concepts de capital circulant et de capital fixe, en dehors du fait que dans les « fonds » il y a les " fonds » naturels ?

Dans " un contexte d'incertitude ", au lieu de « maximiser des gains présents, les économistes devraient chercher à minimiser les regrets futurs que nos actions présentes pourraient susciter ${ }^{18}$. Mais, précisément, on pourrait faire remarquer que, si l'incertitude empêche la maximisation intertemporelle des gains, elle empêche tout autant un calcul optimisateur des regrets futurs. C'est pour cela que, alternativement, s'impose le principe de précaution.

Ces questions affleurent dans le texte de Georgescu-Roegen réédité dans cet ouvrage. D'autres apparaissent plus explicitement. Pour lui, le processus économique est une extension de l'évolution biologique. Jamais il ne lie cette relation aux conditions sociales dans lesquelles

17. Page 13.

18. Page 52. 
elle s'exerce. Voilà un point qui ne marque aucune rupture avec l'idéologie néoclassique : les rapports sociaux sont totalement absents, et, en la matière, absence signifie négation. C'est d'autant plus curieux que Georgescu-Roegen place son analyse dans le cadre d'une co-évolution nature-homme. Or il n'y a pas d'activité économique sans cadre social, sauf, on y revient, à rester dans un schéma de pensée microéconomique et individualiste, tel que celui enseigné par les néoclassiques. Missemer n'en dit mot.

On l'a vu, Georgescu-Roegen récuse la démarche newtonienne mécaniste appliquée à l'économie. Pour cela, il met au même rang l'analyse standard et celle de Marx : " II ne faut pas s'étonner que Karl Marx ait pu défendre avec une insistance sans bornes l'idée que la nature ne joue aucun rôle dans le processus économique puisqu'elle nous offre ces trésors gratuitement ${ }^{19}$. L'erreur contenue dans cette affirmation est tellement grossière qu'on a peine à imaginer que Georgescu-Roegen ait jamais lu Marx et qu'il ne se soit pas contenté de la vulgate diffusée dans tous les manuels de l'économie standard. En effet, Marx a constamment répété que le travail et la terre sont les créateurs de la richesse, le travail créant seul la valeur. " Le travail n'est donc pas l'unique source des valeurs d'usage qu'il produit, de la richesse matérielle. Il en est le père, et la terre la mère, comme dit William Petty ${ }^{20}$. " Ou bien : "Le travail n'est pas la source de toute richesse. La nature est tout autant la source des valeurs d'usage (et c'est bien en cela que consiste la richesse matérielle !) que le travail, qui n'est lui-même que la manifestation d'une force matérielle, de la force de travail humaine ${ }^{21}$. „ Et encore : " La terre peut exercer l'action d'un agent de la production dans la fabrication d'une valeur d'usage, d'un produit matériel,

19. Pages 96-97.

20. Marx K. (1867). Le Capital. In CEuvres, Livre I, Paris, Gallimard, La Pléiade (1965), tome I, p. 571.

21. Marx K. (1875). Critique du programme $d u$ parti ouvrier allemand. In Euvres, tome I, Paris, Gallimard, La Pléiade (1965), p. 1413. disons du blé. Mais elle n'a rien à voir avec la production de la valeur du bléz. " En conséquence, le circuit de la richesse en termes de valeurs d'usage permettant de satisfaire les besoins humains relie le travail et la nature, tandis que le circuit de la valeur relie les humains entre eux et entre eux seulement, car la valeur est une catégorie socio-anthropologique ${ }^{23}$. Ce qui est exact, c'est que, comme le dit Missemer, « toute production de valeur économique se fait par transformation des milieux naturels de manière irréversible ${ }^{24}$, mais cela ne permet pas de dire, comme le font hâtivement nombre d'écologistes, que c'est la nature qui crée la valeur ou une partie de la valeur comme on l'a vu écrire en certains endroits. Et Georgescu-Roegen n'est pas très pas très loin de rejoindre le truisme de la valeur économique intrinsèque de la nature ${ }^{25}:$ " La thermodynamique est au fond une physique de la valeur économique, c'est-à-dire une science qui étudie les qualités physiques qui confèrent en général à certaines choses une valeur pour I'homme $»^{26}$.

Oubli des rapports sociaux et incompréhension de la différence entre richesse et valeur sont le quotidien des économistes standards et des économistes écologiques, et Georgescu-Roegen ne s'en démarque pas, pas plus que Missemer ne le pointe. Pourtant, il aurait été facile de préciser ce que signifie l'idée selon laquelle « la basse entropie devient une condition nécessaire de la valeur économique, sans être toutefois une condition suffisante ${ }^{27}$. Missemer aurait alors vu que cela introduit exactement la même nuance que celle avancée par Marx, ou même par Keynes quand celui-ci distingue le seul facteur de production (le travail) et le cadre environnant

22. Marx K. (1894). Le Capital. Livre III, tome 3 (1974), Paris, Éditions sociales, p. 195, ou dans Euvres, tome II, p. 1430.

23. Voir Harribey J.-M., La richesse, la valeur et l'inestimable, op. cit.

24. Page 84.

25. Pour une critique, voir Harribey J.-M., op. cit.

26. Page 102.

27. Page 23.

Économie Rurale 342/Juillet-Août 2014 • 129 
dans lequel il opère ${ }^{28}$. En l'absence de distinction entre condition nécessaire et condition nécessaire et suffisante, dire que "I'entropie basse est à la base de l'utilité et donc de la valeur économique ${ }^{29}$ risque de renvoyer l'origine de la valeur à l'utilité néoclassique. Et lorsque Missemer reprend l'idée que les schémas de la reproduction de Marx sont aussi critiquables que le paradigme standard " hérité des théories microfondées de l'équilibre général $\aleph^{30}$, il oublie que ces schémas sont une analyse en termes de circuit macro-socio-économique - dont la première ébauche est due à Quesnay - et que cela n'a rien à voir avec une approche en termes d'équilibre de marché, fût-ce général.

Après l'oubli des rapports sociaux et la confusion entre richesse et valeur, le tableau néoclassique se complète avec le dogme de la monnaie-voile : "Une science économique construite sur un échafaudage mécaniste est incapable de traiter des problèmes écologiques indissolublement associés au processus économique, et deuxièmement, [...] on ne peut même pas percevoir ces problèmes si l'on n'écarte pas le voile monétaire et si l'on ne va pas bien au-delà des affaires de marché $\aleph^{31}$. Ici se trouve une vraie difficulté analytique : Georgescu-Roegen a raison de plaider pour une analyse économique prenant en considération les processus de prélèvements sur la nature - ainsi peut-on comprendre une approche réelle -, mais à condition de ne pas retomber sur la démarche classique dite également réelle, mais dont le (l'ir)réalisme consiste à faire comme si nous étions dans une économie de troc sans monnaie.

Missemer note à plusieurs reprises que Georgescu-Roegen fait des processus économiques une extension de l'évolution endosomatique, tandis que le renouvellement et la circulation des élites dirigeantes

28. Keynes J.-M. (1936). Théorie générale de l'emploi, de l'intérêt et de la monnaie. Paris, Payot, 1969, p. 223.

29. Page 39.

30. Page 19.

31. Page 100. sont attribués à l'évolution exosomatique ${ }^{32}$. $N^{\prime} y$ aurait-il pas là une nouvelle raison de s'interroger si le déterminisme technique venait s'immiscer dans un cadre théorique qui se veut résolument non mécaniste?

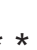

Il n'empêche, le livre d'Antoine Missemer est d'un très grand intérêt. D'abord, il donne de bonnes raisons pour s'engager en faveur d'une reconversion bioéconomique, voire en faveur du développement durable, mais sans " optimisme naïf » et à condition que cela ne prenne pas " la forme d'une aspiration à un salut écologique absolu ", car " l'échelle temporelle humaine est plus courte que l'échelle géologique, mais elle est aussi plus longue que l'échelle des processus économiques du quotidien $1^{33}$. Ensuite, il donne aux chercheurs une excellente synthèse d'une œuvre complexe consacrée à une problématique qui se trouve être au carrefour de la physique, de la biologie, de la géologie et de l'économie, sinon des sciences sociales. Enfin, last but not least, les économistes qui ignoreraient jusqu'au nom de Georgescu-Roegen n'auront plus d'excuse. Laissons-lui donc le dernier mot : " La masse peut être produite en partant de l'énergie et de la masse, comme dans la réaction qui mène au plutonium, jamais de l'énergie seule. L'énergie seule ne peut pas être transformée en masse même dans les étoiles où les conditions sont extrêmement favorables à la fusion des particules élémentaires. Et le hic est que sans masse, il n'y a pas de protons, et sans protons, il n'y a pas de matière. La preuve en est que les théories courantes placent l'origine des éléments chimiques de l'univers dans l'hydrogène et, peut-être, dans I'hélium aussi et non pas dans les photons seulement $»^{34}$.

\section{Jean-Marie HARRIBEY ${ }^{35}$} Université Bordeaux IV
32. Page 95 , note 5 .

33. Page 80.

34. Page 111.

35. http://harribey.u-bordeaux4.fr ; http://alternatives-economiques.fr/blogs/harribey. 